\title{
Validation and Enhancement of the Clinicopathological Melanoma Nomogram via Incorporation of a Molecular Marker in the Primary Tumor
}

\author{
SAMER A. NAFFOUJE ${ }^{1}$, RAND NAFFOUJE ${ }^{2}$, JINSONG CHEN $^{3}$ and GEORGE I. SALTI ${ }^{4}$ \\ ${ }^{1}$ Department of General Surgery, University of Illinois Hospital and Health Sciences System, Chicago, IL, U.S.A.; \\ ${ }^{2}$ Department of Internal Medicine, Louis Weiss Memorial Hospital, Chicago, IL, U.S.A.; \\ ${ }^{3}$ Department of Biostatistics, University of Illinois at Chicago College of Medicine, Chicago, IL, U.S.A.; \\ ${ }^{4}$ Division of Surgical Oncology, University of Illinois at Chicago Hospital \\ and Health Sciences System, Chicago, IL, U.S.A.
}

\begin{abstract}
Background/Aim: To validate the melanoma nomogram and improve its function in prediction of nodal dissemination by incorporating a molecular marker in the model. Microphthalmia transcription factor (MITF) is an important regulator of melanocyte homeostasis and differentiation. We have shown that the grade of MITF expression in primary melanoma cells can serve as a predictor of nodal status. Many efforts to identify the nodal spread in cutaneous melanoma using non-invasive means have been recently undertaken. A nomogram was developed by Memorial Sloan Kettering Cancer Center (MSKCC) based on clinicopathological features of the primary melanoma to predict the nodal status. In this study, we applied the same nomogram for external validation. Then, we added MITF as an independent predictive factor, and assessed its impact on the nomogram's accuracy in prediction of the nodal spread. Materials and Methods: We included 171 patients with melanoma with available tumor specimens, and used MITF staining grade of $\geq 50 \%$ as a pathological characteristic of the primary tumor in addition to age, location, thickness, Clark level, and ulceration, as reported by MSKCC. Results: Upon comparison of receiver operating curves, we confirmed the external validation of the melanoma nomogram, in accordance with the MSKCC curves [area under the curve (AUC) 0.742 vs. 0.650]. Addition of MITF $\geq 50 \%$ as an independent factor in the analysis improved the model fit significantly $(A U C=0.825$ vs. $0.742 ; p<0.0001)$. Conclusion:
\end{abstract}

Correspondence to: Samer Naffouje, MD, University of Illinois at Chicago Hospital and Health Sciences System, 840 S Wood Street, Suite 376 CSN, Chicago, IL 60612, U.S.A. Tel: +1 3129969365, Fax: +1 3124133722, e-mail: snaffouj@uic.edu

Key Words: Microphthalmia transcription factor, melanoma, nomogram.
The nomogram described by MSKCC is a valuable tool in predicting sentinel lymph node involvement in primary cutaneous melanoma. Addition of MITF $\geq 50 \%$ into the logistic regression analysis significantly improves the accuracy of the melanoma nomogram in prediction of regional nodal spread.

Microphthalmia transcription factor (MITF) is the most prominent member of the MIT proteins, a family of basic helix-loop-helix proteins that bind to CANNTG DNA promoter E-box, and regulate cellular differentiation, mitosis, and apoptosis cascades (1). The role of MITF is evident in homeostasis of certain human cell lines such as osteocytes, mast cells, and most importantly, melanocytes (1), where it functions as a downstream factor in the Ras-activated factor$\mathrm{B} /$ mitogen-activated protein kinase/extracellular signalregulated kinase (BRAF/MEK/ERK) pathway (2); BRAFmutant malignant melanocytes have decreased levels of MITF, whereas in melanoma cells treated with BRAF inhibitors, the MITF level was restored and cell-cycle arrest and apoptosis induced (2). Therefore, the presence of MITF in melanoma cells has been judged a favorable biological marker. Indeed, we were able to demonstrate that increased MITF expression in primary melanoma was associated with less aggressive phenotypes (3), decreased tumor growth (4), and improved overall and disease-free survival (5). Moreover, we recently showed that MITF expression in primary melanoma carries a predictive value for sentinel lymph node (SLN) metastasis (6).

Nodal dissemination is currently considered the most important prognosticator in malignant melanoma (7), with 5year survival decreasing by two- to three-fold when lymph node involvement is detected (8). In current practice, histology of the SLN remains the gold standard for assessing locoregional dissemination. Recently, there has been a growing trend for predicting the SLN status using noninvasive approaches. Most importantly, a nomogram was established by the Memorial Sloan-Kettering Cancer Center 
(MSKCC) to predict nodal metastasis based on clinical and pathological characteristics of the primary melanoma tumor (9). Herein, we aimed to apply and externally validate this nomogram in our melanoma population at the University of Illinois at Chicago Medical Center (UICMC), and assess whether the incorporation of MITF increases the nomogram's accuracy in prediction of nodal status.

\section{Materials and Methods}

UICMC tissue bank included specimens from patients diagnosed and treated for cutaneous melanoma between 1976 and 2006. Collected demographic and histopathological data included patient age, melanoma location, thickness (Clark and Breslow levels), and ulceration per MSKCC's nomogram.

Four- $\mu$ m-thick sections of the primary tumor were used for immunocytochemical staining using mouse monoclonal IgG1 microphthalmia antibody Ab-2 (Neomarkers, Union City, CA, USA). Antigen retrieval was accomplished with $1 \mathrm{~mm}$ EDTA buffer. We used $1 \%$ dried skim milk to block nonspecific binding before incubation with the primary antibody for $2 \mathrm{~h}$ (1:25 dilution). Aminoethylcarbazine was used as a chromogen. All the slides were evaluated by the same examiner. The stained slides were graded according to the percentage of cells whose nuclei stained positive for MITF in the entire fields of the slide. Cytoplasmic staining was excluded from the analysis. MITF staining cut-off of $\geq 50 \%$ of the melanoma cells per slide (MITF $\geq 50 \%$ ) was used as an additional characteristic of the primary tumor based on our previous study (6).

We used the logistic regression model to fit the data without considering MITF in order to validate the MSKCC nomogram in our patient population. We then added $\mathrm{MITF} \geq 50 \%$ to the model to assess its impact on the nomogram's predictability of the nodal status. Likelihood ratio test was conducted to test the effect of the addition of $\mathrm{MITF} \geq 50$ into the model.

\section{Results}

This study included 171 patients with primary cutaneous melanoma whose tumors were eligible for proper specimen collection into our tumor bank between 1976-2006. All patients consented to specimen collection under an Institutional Review Board protocol \#2002-0540. The tumor bank included patients who underwent nodal sampling using sentinel node biopsies or elective node dissection prior to the sentinel node era, as well as patients who received planned nodal basin dissection for palpable disease. Their mean age was $55.5 \pm 15.8$ years (range $=17-89$ years). Mean tumor thickness was $2.55 \pm 2.17 \mathrm{~mm}$ (range $=10.43-22 \mathrm{~mm}$ ). A total of 101 of our patients were females $(59.06 \%)$. Histopathological characteristics of our patients with melanoma are summarized in Table I.

Firstly, we used the MSKCC logistic regression model with coefficients locked-down then fit to our data. The model has the form:

Logit (probability of positive nodes):

$=\beta_{0}+\beta_{1}$ Head/Neck $+\beta_{2}$ Trunk $+\beta_{3}$ Level IV $+\beta_{4}$ Level

$V+\beta_{5}$ Ulceration $+f_{1}$ (age) $+f_{2}$ (thickness) (Eq. 1)
Table I. Summary of the characteristics of our 171 patients with melanoma.

\begin{tabular}{|c|c|}
\hline Characteristic & Value \\
\hline Female, n (\%) & $101(59.06 \%)$ \\
\hline Mean age $\pm S D$, years & $55.5 \pm 15.8$ \\
\hline \multicolumn{2}{|l|}{ Location, n (\%) } \\
\hline Head \& neck & $23(13.5 \%)$ \\
\hline Upper extremity & $62(36.3 \%)$ \\
\hline Trunk & $57(33.3 \%)$ \\
\hline Lower extremity & $27(15.8 \%)$ \\
\hline Perineal & $1(0.6 \%)$ \\
\hline Missing & $1(0.6 \%)$ \\
\hline \multicolumn{2}{|l|}{ Clark level, n (\%) } \\
\hline 1 & $2(1.2 \%)$ \\
\hline 2 & $4(2.3 \%)$ \\
\hline 3 & $35(20.5 \%)$ \\
\hline 4 & $121(70.8 \%)$ \\
\hline 5 & $8(4.7 \%)$ \\
\hline Missing data & $1(0.6 \%)$ \\
\hline \multicolumn{2}{|l|}{ Breslow's thickness, n (\%) } \\
\hline$<1 \mathrm{~mm}$ & $8(4.68 \%)$ \\
\hline $1.00-2 \mathrm{~mm}$ & $79(46.19 \%)$ \\
\hline $2.01-4 \mathrm{~mm}$ & $67(39.18 \%)$ \\
\hline$>4 \mathrm{~mm}$ & $17(9.95 \%)$ \\
\hline \multicolumn{2}{|l|}{ Histology, n (\%) } \\
\hline Superficial spreading & $73(42.7 \%)$ \\
\hline Nodular & $62(36.3 \%)$ \\
\hline Lentigo maligna & $11(6.4 \%)$ \\
\hline Acral & $169.4 \%)$ \\
\hline Dysplastic & $8(4.7 \%)$ \\
\hline Missing data & $1(0.6 \%)$ \\
\hline \multicolumn{2}{|l|}{ Ulceration } \\
\hline Absent & $110(64.3 \%)$ \\
\hline Present & $60(35.1 \%)$ \\
\hline Missing data & $1(0.6 \%)$ \\
\hline \multicolumn{2}{|l|}{ MITF staining, $\mathrm{n}(\%)$} \\
\hline $\mathrm{MITF}<50 \%$ & $100(58.5 \%)$ \\
\hline $\mathrm{MITF} \geq 50 \%$ & $71(41.5 \%)$ \\
\hline \multicolumn{2}{|l|}{ Node pathology, n (\%) } \\
\hline Negative & $119(69.6 \%)$ \\
\hline Positive & $52(30.4 \%)$ \\
\hline
\end{tabular}

SD: Standard deviation; MITF: microphthalmia transcription factor.

where $f_{1}$ (age) and $f_{2}$ (thickness) are nonlinear functions modeled by the restricted cubic splines to accommodate potential nonlinear effects for age and thickness. By plotting the receiver operating curves of logistic regression (Eq. 1), the MSKCC nomogram demonstrated an area under the curve (AUC) of 0.650, whereas fitting the coefficients to our data gave an AUC of 0.742 (Figure 1A).

We then used the same logistic regression model with the addition of MITF $\geq 50 \%$ as a predictor. The model had the form: Logit (probability of positive nodes):

$=\beta_{0}+\beta_{1}$ Head/Neck $+\beta_{2}$ Trunk $+\beta_{3}$ Level IV $+\beta_{4}$ Level $V+\beta_{5}$ Ulceration $+f_{1}$ (age) $+f_{2}$ (thickness) $+\beta_{6}$ $M I T F \geq 50 \%$ (Eq. 2). 
In the model fit, the estimated coefficient for MITF $\geq 50 \%$ was -2.2035 ( $p=0.0002$ ), indicating that patients with MITF $\geq 50 \%$ are significantly less likely to have nodal disease compared to those with MITF $<50 \%$. Likelihood ratio test was conducted to test the effect of the addition of MITF $\geq 50 \%$ to the model. The results indicate that the inclusion of MITF $\geq 50 \%$ improved the model fit significantly $(p<0.001)$. By comparison of receiver operating curves (Figure 1C), the addition of MITF to the model raised the AUC from 0.742 to 0.825 , significantly improving the accuracy of the nomogram in predicting the lymph node involvement. Figure 2 demonstrates our nomogram scoring table with a projection on predicted probability values.

\section{Discussion}

Involvement of regional lymph nodes remains the single most important prognostic factor in cutaneous melanoma, where survival is reduced from $89-95 \%$ and $45-79 \%$ in stages I and II, respectively, to $24-70 \%$ in stage III when lymph node metastasis is evident (8). Histological evaluation of the SLN remains the gold standard in nodal staging for cutaneous melanoma in the current oncologic practice.

The indications for SLN biopsy continue to be somewhat uninformed. It is clearly recommended by the National Comprehensive Cancer Network that patients with melanoma undergo a SLN biopsy when tumor thickness exceeds $1 \mathrm{~mm}$ (10). Patients with thin melanomas $(<1 \mathrm{~mm})$ carry a risk as low as $2-5 \%$ of nodal spread $(11,12)$, thus making patient selection for SLN biopsy a topic of high importance in this group. Current evidence proposes SLN biopsy for patients whose tumor thickness ranges between 0.76 and $1 \mathrm{~mm}$ if they demonstrate "adverse features" such as lymphovascular invasion, age $<40$ years, significant vertical growth phase, increased mitotic rate, positive deep margins, and Clark's level IV or higher (8). Thick melanomas $(>4 \mathrm{~mm})$ with clinically negative lymph nodes were associated with SLN positivity in $30-40 \%$ of cases $(13,14)$. However, the high likelihood of systemic spread in patients with thick melanomas renders the survival benefit from lymph node dissection somewhat debatable (15). Moreover, the role of completion of lymph node dissection in the face of a positive SLN in terms of survival benefit is yet to be clarified, and is currently a trending theme in this field.

According to the above, multiple approaches have been undertaken to stratify patients with melanoma into risk categories for nodal metastasis in an attempt to waive the unnecessary risk of surgical procedures in those who bear a very low risk of nodal spread.

The nomogram developed by the MSKCC (9), a tertiary cancer center in the U.S., was a pioneer endeavor in this regard. This nomogram was constructed using five commonly reported characteristics of primary melanoma
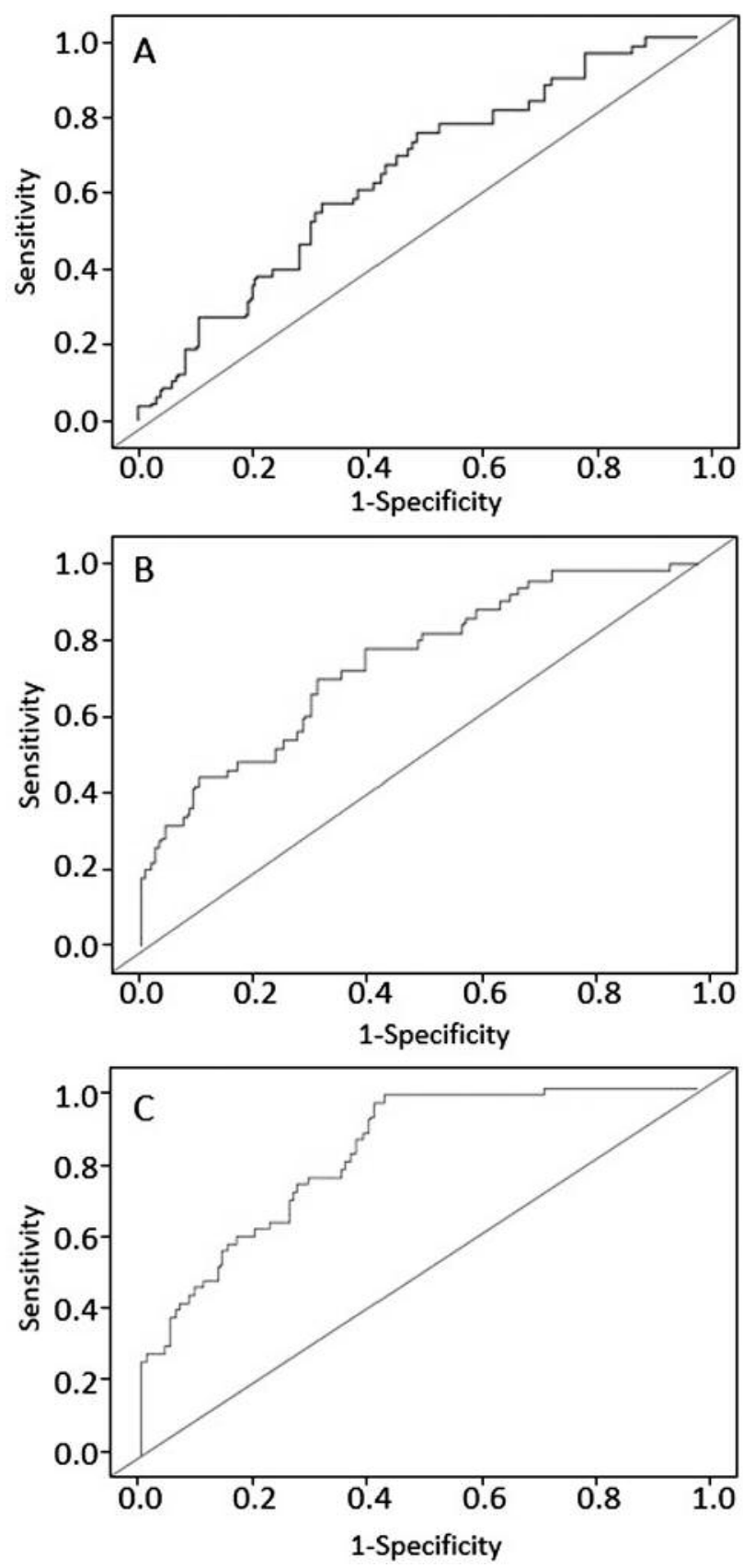

Figure 1. Receiver operating curves. A: Results of the Memorial Sloan Kettering Cancer Center nomogram with coefficients locked-down; area under the curve $(A U C)=0.650 . B$ : Results of the University of Illinois at Chicago Medical Center nomogram with coefficients fit into the data; $A U C=0.742$; microphthalmia transcription factor (MITF) was not considered in the model. C: Results of the University of Illinois at Chicago Medical Center nomogram after fitting MITF $\geq 50 \%$ into the model; $A U C=0.825(p<0.001)$.

tumors: thickness, ulceration, Clark level, age, and tumor location. The model that was generated based on the simultaneous interaction of these variables was found to be 

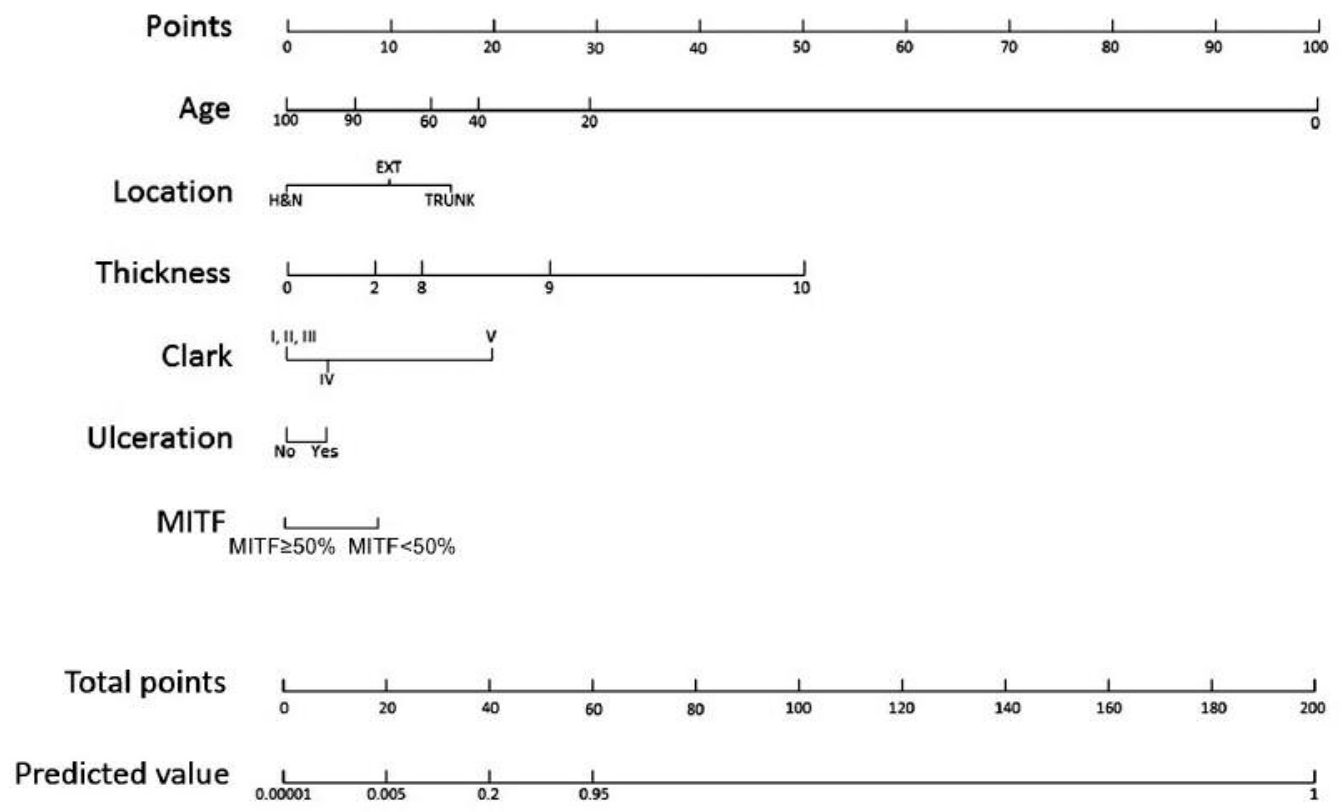

Figure 2. University of Illinois at Chicago Medical Center melanoma nomogram score table. MITF: Microphthalmia transcription factor.

quantitatively more discriminating than the American Joint Committee on Cancer staging system in prediction of regional melanoma spread (7).

In a parallel effort, our group focused on the expression of MITF, a favorable molecular marker in cutaneous melanoma, in the primary tumors as a predictive factor of nodal involvement. Certainly, we showed that patients with higher MITF expression were less likely to have nodal spread (6). More specifically, we demonstrated in that report that none of the patients with MITF $\geq 50 \%$ had positive SLN. Given this significant sensitivity, MITF expression of $\geq 50 \%$ was selected as the optimized cut-off for this marker as a predictor in the nomogram model. Herein, we proceeded to apply and externally validate the MSKCC nomogram in our patient population, and assess whether the addition of MITF would improve the nomogram's predictability of the nodal status.

As demonstrated above, the MSKCC nomogram was a valuable tool in predicting nodal involvement in our population, with an $\mathrm{AUC}=0.65$ when coefficients were locked-down, and $\mathrm{AUC}=0.742$ after fitting. It is currently agreed that an AUC of a receiver operating curves $>0.6$ provides reasonable discrimination, AUC $>0.7$ represents good discrimination, and AUC $>0.8$ represents excellent discrimination (16). Notably, there were some differences in the magnitude of regression coefficients between our UICMC and MSKCC nomograms which could be explained by the intrinsic differences between the two populations, but the signs of significant coefficients were in agreement. Inclusion of patients with clinically positive nodal disease increased the rate of node positivity in our population compared to the usual rates detected by SLN biopsies. However, this would not interfere with the function of the nomogram in detecting a correlation between the factor set and nodal status, regardless of the clinical findings.

The MSKCC melanoma nomogram was previously validated by European groups (17-19) with concordance indices of $>0.8$. The current study represents the first external validation of this nomogram in a tertiary care center in the U.S.

Given the significance of MITF expression in prediction of nodal involvement, the regression analysis was reapplied after incorporation of the molecular marker into the model fit. Evidently, the accuracy of the nomogram increased to AUC $=0.825 \quad(p<0.001)$.

The purpose of developing nomograms in medical practice, and particularly in oncology, is to better predict certain outcomes in individual patients. Similar nomograms have been evolving for prediction of prostate cancer recurrence $(20,21)$ and non-SLN involvement in breast cancer $(22,23)$, with promising and reproducible results .

The melanoma nomogram developed here is an effort toward the establishment of accurate selection criteria for SLN sampling, in order to avoid redundancy and the complications of surgical interventions in patients with a low risk of disease spread. Nonetheless, the main criticism of nomogram-based predictions remains about their practical application in the clinical setting. Generally, the MSKCC melanoma nomogram can be used to decide when 
not to perform a SLN biopsy based on its negative predictive value (NPV).

In order to shed further light on the influence of MITF on the nomogram's precision, we tested both the MSKCC and UICMC nomograms on our patient population to understand how they would have affected the surgical decision. We used a pre-set predicted probability cut-off of $<9 \%$ as reported by previous validations (18) to avoid the data-driven overestimation of sensitivity and specificity (24). Results are presented in Tables II and III.

It is evident that the addition of MITF into the model significantly improved the precision in regards to sensitivity, specificity, NPV, and error rates. It was noted that the discrepancy between the nomograms increased as the tumors become thicker, since the MSKCC nomogram relies heavily on the tumor's thickness (highest $\beta$-coefficient), whereas our nomogram relies more on MITF status.

The refinement in the nomogram is attributable to the predictive value of MITF for nodal spread. This, in turn, stems from the critical biological role of MITF in melanocyte homeostasis by controlling proliferation and triggering cellcycle exit (25). Recent reports investigating the molecular mechanisms of melanoma development documented that high levels of MITF expression exert an antiproliferative activity in melanoma cells (26), whereas low levels of MITF protein were found in invasive melanoma cells (27). However, the majority of melanoma cells tend to maintain a low basal expression of this protein (28). This ambivalent function of MITF made it difficult to use for targeted therapy. Instead, detection of low levels of MITF or gene amplification have been utilized as diagnostic approaches for this malignancy (29-35). Numerous studies addressed the correlation of diminutive expression of MITF in primary melanoma with poor prognosis and clinical disease progression $(4,5,26,27$, $32,34,36)$. In addition, MITF is suggested to be involved in BRAF-targeted therapy resistance. Melanoma resistance to BRAF inhibitors occurs due to a cAMP response element binding protein escape after RAF/MEK inhibition via intracellular signaling network, leading to activation of downstream transcription factors such as fibroblast osteogenic sarcoma (c-FOS), nuclear receptor subfamily 4 group A members 1 and 2 (NR4A1, NR4A2) and MITF (37). Another extracellular mechanism was suggested via WNT and secreted frizzled-related protein 2 (sFRP2), proteins secreted by aged fibroblasts, which diminish $\beta$-catenin and MITF levels in melanocytes, resulting in loss of DNA-apurinic/apyrimidinic site phosphomonoester-lyase endonuclease (APE1), a major redox effector. Subsequently, melanoma cells fail to respond to DNA damage and become more resistant to BRAF-targeted therapy (38).

Based on the above, it is clear that MITF is part of a multitude of melanoma development pathways, which makes it an interesting focus of various clinical applications,
Table II. Comparative analysis of Memorial Sloan Kettering Cancer Center (MSKCC) and University of Illinois at Chicago Medical Center (UICMC) nomograms in our patient population at a predicted probability cut-off of $<9 \%$. ( $N=171$, one patient had missing data and was excluded from this analysis).

\begin{tabular}{llccr}
\hline Nomogram & & Node + & Node - & Total \\
\hline \multirow{2}{*}{ MSKCC } & Nomogram + & 38 & 76 & 115 \\
& Nomogram - & 14 & 42 & 55 \\
& Total & 52 & 118 & 170 \\
UICMC & Nomogram + & 46 & 66 & 112 \\
& Nomogram - & 6 & 52 & 58 \\
& Total & 52 & 118 & 170 \\
\hline
\end{tabular}

Table III. Comparison of precision parameters for Memorial Sloan Kettering Cancer Center (MSKCC) and University of Illinois at Chicago Medical Center (UICMC) nomograms for our patient population at a predicted probability cut-off of $<9 \%$. ( $N=171$, one patient had missing data and was excluded from this analysis).

\begin{tabular}{lcc}
\hline & $\begin{array}{c}\text { MSKCC } \\
\text { nomogram results }\end{array}$ & $\begin{array}{c}\text { UICMC } \\
\text { nomogram results }\end{array}$ \\
\hline Sensitivity & $73.08 \%$ & $88.46 \%$ \\
Specificity & $35.59 \%$ & $44.07 \%$ \\
NPV & $76.36 \%$ & $89.65 \%$ \\
Reduction rate & $32.35 \%$ & $34.12 \%$ \\
Error rate & $8.24 \%$ & $3.53 \%$ \\
\hline
\end{tabular}

NPV: Negative predictive value. Reduction rate: Sentinel node biopsy (SNB) reduction rate $=[\mathrm{TN}+\mathrm{FN}] /[\mathrm{TN}+\mathrm{FN}+\mathrm{TP}+\mathrm{FP}]$, which represents the proportion of patients classified as negative and thus potentially selected by the nomogram for being spared the SNB procedure. Error rate: error rate $=\mathrm{FN} /[\mathrm{FN}+\mathrm{TN}+\mathrm{TP}+\mathrm{TN}]$, which is the proportion of patients erroneously classified as negative by the nomogram (these patients would be selected by the nomogram to be spared the SNB procedure although they do harbor SN metastatic disease). FN/FP and TN/TP: False/true and negative/positive.

including diagnosis, therapy, and outcome prediction. In our study, we aimed to utilize this factor to improve our ability to predict regional melanoma spread. The shortcomings of this study stem from the heterogeneous nature of our population, which was recruited over a long period of time. Some patients underwent nodal sampling using the SLN biopsy, some had elective node dissection prior to the SLN era, and others underwent a complete nodal basin dissection for palpable nodes. This could explain, at least partly, the relatively high rate of positive nodes in our population. However, given the cross-sectional nature of the statistical analysis, using this population for validation remains suitable, and the statistical analysis remains appropriate. 
Currently, only $15-20 \%$ of all SLN biopsies for melanoma are reported to be positive, but much lower rates are reported for patients with melanomas $<1.2 \mathrm{~mm}$ thick according to the Multicenter Selective lymphadenectomy Trial group (39) and others $(40,41)$. Moreover, SLN false-negativity rates, based on the contemporary indications, are reported by numerous multicenter trials to be $4-14.4 \%$ (42-48). This suggests that SLN biopsy might be overused nowadays, and that its indications require refinement.

Therefore, our group supports the incorporation of the MSKCC nomogram into clinical practice. The addition of primary tumor prognostic factors, such as MITF, enhances the nomogram's accuracy and should be considered, taking into account its generalizability for uniform application and further validation.

\section{Conclusion}

The MSKCC melanoma nomogram is a valuable tool for predicting nodal spread in cutaneous melanoma, and has been externally validated in a variety of centers and settings, including ours. In our opinion, it constitutes a valid effort toward refining the selection criteria for SLN biopsy in patients with melanoma. Inclusion of MITF $\geq 50 \%$ as a favorable prognostic factor in the model significantly increases its accuracy in predicting nodal spread.

\section{Acknowledgements}

Special thanks to Dr. Michael Kattan and his team for providing the original nomogram formula, and for their assistance during the process of statistical analysis.

\section{References}

1 Hershey CL and Fisher DE: MITF and TFE3: Members of a bHLH-zip transcription factor family essential for osteoclast development and function. Bone 34(4): 689-696, 2004.

2 Haq R, Shoag J, Andreu-Perez P, Yokoyama S, Edelman H, Rowe GC, Frederick DT, Hurley AD, Nellore A, Kung AL, Wargo JA, Song JS, Fisher DE, Arany Z and Widlund HR: Oncogenic BRAF regulates oxidative metabolism via PGC1alpha and mitf. Cancer Cell 23(3): 302-315, 2013.

3 Lekmine F, Chang CK, Sethakorn N, Das Gupta TK and Salti GI: Role of microphthalmia transcription factor (MITF) in melanoma differentiation. Biochem Biophys Res Commun 354(3): 830-835, 2007.

4 Selzer E, Wacheck V, Lucas T, Heere-Ress E, Wu M, Weilbaecher KN, Schlegel W, Valent P, Wrba F, Pehamberger H, Fisher D and Jansen B: The melanocyte-specific isoform of the microphthalmia transcription factor affects the phenotype of human melanoma. Cancer Res 62(7): 2098-2103, 2002.

5 Salti GI, Manougian T, Farolan M, Shilkaitis A, Majumdar D and Das Gupta TK: Micropthalmia transcription factor: A new prognostic marker in intermediate-thickness cutaneous malignant melanoma. Cancer Res 60(18): 5012-5016, 2000.
6 Naffouje S, Naffouje R, Bhagwandin S and Salti GI: Microphthalmia transcription factor in malignant melanoma predicts occult sentinel lymph node metastases and survival. Melanoma Res 25(6): 496-502, 2015.

7 Balch CM, Buzaid AC, Soong SJ, Atkins MB, Cascinelli N, Coit DG, Fleming ID, Gershenwald JE, Houghton A, Jr., Kirkwood JM, McMasters KM, Mihm MF, Morton DL, Reintgen DS, Ross MI, Sober A, Thompson JA and Thompson JF: Final version of the American Joint Committee on Cancer Staging System for Cutaneous Melanoma. J Clin Oncol 19(16): 3635-3648, 2001.

8 Coit DG, Thompson JA, Andtbacka R, Anker CJ, Bichakjian CK, Carson WE, 3rd, Daniels GA, Daud A, Dimaio D, Fleming MD, Gonzalez R, Guild V, Halpern AC, Hodi FS, Jr., Kelley MC, Khushalani NI, Kudchadkar RR, Lange JR, Martini MC, Olszanski AJ, Ross MI, Salama A, Swetter SM, Tanabe KK, Trisal V, Urist MM, McMillian NR, Ho M and National Comprehensive Cancer N: Melanoma, version 4.2014. J Natl Compr Canc Netw 12(5): 621-629, 2014.

9 Wong SL, Kattan MW, McMasters KM and Coit DG: A nomogram that predicts the presence of sentinel node metastasis in melanoma with better discrimination than the American Joint Committee on Cancer Staging System. Ann Surg Oncol 12(4): 282-288, 2005.

10 Amersi F and Hansen NM: The benefits and limitations of sentinel lymph node biopsy. Curr Treat Options Oncol 7(2): 141$151,2006$.

11 Thompson JF and Shaw HM: Sentinel node mapping for melanoma: Results of trials and current applications. Surg Oncol Clin N Am 16(1): 35-54, 2007.

12 Stadelmann WK: The role of lymphatic mapping and sentinel lymph node biopsy in the staging and treatment of melanoma. Clin Plast Surg 37(1): 79-99, 2010.

13 Ferrone CR, Panageas KS, Busam K, Brady MS and Coit DG: Multivariate prognostic model for patients with thick cutaneous melanoma: Importance of sentinel lymph node status. Ann Surg Oncol 9(7): 637-645, 2002.

14 Gershenwald JE, Mansfield PF, Lee JE and Ross MI: Role for lymphatic mapping and sentinel lymph node biopsy in patients with thick $(>$ or $=4 \mathrm{~mm}$ ) primary melanoma. Ann Surg Oncol 7(2): 160-165, 2000.

15 Fontaine D, Parkhill W, Greer W and Walsh N: Partial regression of primary cutaneous melanoma: Is there an association with sub-clinical sentinel lymph node metastasis? Am J Dermatopathol 25(5): 371-376, 2003.

16 Hanley JA and McNeil BJ: A method of comparing the areas under receiver operating characteristic curves derived from the same cases. Radiology 148(3): 839-843, 1983.

17 Pinero A, Canteras M, Ortiz E, Martinez-Barba E and Parrilla P: Validation of a nomogram to predict the presence of sentinel lymph node metastases in melanoma. Ann Surg Oncol 15(10): 2874-2877, 2008.

18 Pasquali S, Mocellin S, Campana LG, Vecchiato A, Bonandini E, Montesco MC, Santarcangelo S, Zavagno G, Nitti D and Rossi CR: Maximizing the clinical usefulness of a nomogram to select patients candidate to sentinel node biopsy for cutaneous melanoma. Eur J Surg Oncol 37(8): 675-680, 2011.

19 Woods JF, De Marchi JA, Lowery AJ and Hill AD: Validation of a nomogram predicting sentinel lymph node status in melanoma in an irish population. Ir J Med Sci 184(4): 769-773, 2015 . 
20 Greene KL, Meng MV, Elkin EP, Cooperberg MR, Pasta DJ, Kattan MW, Wallace K and Carroll PR: Validation of the kattan preoperative nomogram for prostate cancer recurrence using a community based cohort: Results from Cancer of the Prostate Strategic Urological Research Endeavor (CAPSURE). J Urol 171(6 Pt 1): 2255-2259, 2004.

21 Yanke BV, Gonen M, Scardino PT and Kattan MW: Validation of a nomogram for predicting positive repeat biopsy for prostate cancer. J Urol 173(2): 421-424, 2005.

22 Smidt ML, Kuster DM, van der Wilt GJ, Thunnissen FB, Van Zee KJ and Strobbe LJ: Can the Memorial Sloan-Kettering Cancer Center nomogram predict the likelihood of nonsentinel lymph node metastases in breast cancer patients in the netherlands? Ann Surg Oncol 12(12): 1066-1072, 2005.

23 Lambert LA, Ayers GD, Hwang RF, Hunt KK, Ross MI, Kuerer HM, Singletary SE, Babiera GV, Ames FC, Feig B, Lucci A, Krishnamurthy $S$ and Meric-Bernstam F: Validation of a breast cancer nomogram for predicting nonsentinel lymph node metastases after a positive sentinel node biopsy. Ann Surg Oncol 13(3): 310-320, 2006.

24 Leeflang MM, Moons KG, Reitsma JB and Zwinderman AH: Bias in sensitivity and specificity caused by data-driven selection of optimal cutoff values: Mechanisms, magnitude, and solutions. Clin Chem 54(4): 729-737, 2008.

25 Opdecamp K, Nakayama A, Nguyen MT, Hodgkinson CA, Pavan WJ and Arnheiter H: Melanocyte development in vivo and in neural crest cell cultures: Crucial dependence on the MITF basic-helix-loop-helix-zipper transcription factor. Development 124(12): 2377-2386, 1997.

26 Wellbrock C and Marais R: Elevated expression of MITF counteracts B-RAF-stimulated melanocyte and melanoma cell proliferation. J Cell Biol 170(5): 703-708, 2005.

27 Hoek KS, Eichhoff OM, Schlegel NC, Dobbeling U, Kobert N, Schaerer L, Hemmi S and Dummer R: In vivo switching of human melanoma cells between proliferative and invasive states. Cancer Res 68(3): 650-656, 2008.

28 Kordass T, Weber CE, Oswald M, Ast V, Bernhardt M, Novak D, Utikal J, Eichmuller SB and Konig R: SOX5 Is involved in balanced MITF regulation in human melanoma cells. BMC Med Genomics 9: 10, 2016.

29 King R, Weilbaecher KN, McGill G, Cooley E, Mihm M and Fisher DE: Microphthalmia transcription factor. A sensitive and specific melanocyte marker for melanomadiagnosis. Am J Pathol 155(3): 731-738, 1999.

30 Miettinen M, Fernandez M, Franssila K, Gatalica Z, Lasota J and Sarlomo-Rikala M: Microphthalmia transcription factor in the immunohistochemical diagnosis of metastatic melanoma: Comparison with four other melanoma markers. Am J Surg Pathol 25(2): 205-211, 2001.

31 Chang KL and Folpe AL: Diagnostic utility of microphthalmia transcription factor in malignant melanoma and other tumors. Adv Anat Pathol 8(5): 273-275, 2001.

32 Levy C, Khaled M and Fisher DE: MITF: Master regulator of melanocyte development and melanoma oncogene. Trends Mol Med 12(9): 406-414, 2006.

33 Garraway LA and Sellers WR: Lineage dependency and lineagesurvival oncogenes in human cancer. Nat Rev Cancer 6(8): 593602, 2006.

34 Garraway LA, Widlund HR, Rubin MA, Getz G, Berger AJ, Ramaswamy S, Beroukhim R, Milner DA, Granter SR, Du J,
Lee C, Wagner SN, Li C, Golub TR, Rimm DL, Meyerson ML, Fisher DE and Sellers WR: Integrative genomic analyses identify MITF as a lineage survival oncogene amplified in malignant melanoma. Nature 436(7047): 117-122, 2005.

35 Zhuang L, Lee CS, Scolyer RA, McCarthy SW, Zhang XD, Thompson JF and Hersey P: MCL-1, BCL-xl and STAT3 expression are associated with progression of melanoma whereas BCL-2, AP-2 and MITF levels decrease during progression of melanoma. Mod Pathol 20(4): 416-426, 2007.

36 Palmieri G, Capone M, Ascierto ML, Gentilcore G, Stroncek DF, Casula M, Sini MC, Palla M, Mozzillo N and Ascierto PA: Main roads to melanoma. J Transl Med 7: 86, 2009.

37 Johannessen CM, Johnson LA, Piccioni F, Townes A, Frederick DT, Donahue MK, Narayan R, Flaherty KT, Wargo JA, Root DE and Garraway LA: A melanocyte lineage program confers resistance to map kinase pathway inhibition. Nature 504(7478): 138-142, 2013.

38 Kaur A, Webster MR, Marchbank K, Behera R, Ndoye A, Kugel CH, 3rd, Dang VM, Appleton J, O'Connell MP, Cheng P, Valiga AA, Morissette R, McDonnell NB, Ferrucci L, Kossenkov AV, Meeth K, Tang HY, Yin X, Wood WH, 3rd, Lehrmann E, Becker KG, Flaherty KT, Frederick DT, Wargo JA, Cooper ZA, Tetzlaff MT, Hudgens C, Aird KM, Zhang R, Xu X, Liu Q, Bartlett E, Karakousis G, Eroglu Z, Lo RS, Chan M, Menzies AM, Long GV, Johnson DB, Sosman J, Schilling B, Schadendorf D, Speicher DW, Bosenberg M, Ribas A and Weeraratna AT: SFRP2 in the aged microenvironment drives melanoma metastasis and therapy resistance. Nature 532(7598): 250-254, 2016.

39 Morton DL, Thompson JF, Cochran AJ, Mozzillo N, Nieweg OE, Roses DF, Hoekstra HJ, Karakousis CP, Puleo CA, Coventry BJ, Kashani-Sabet M, Smithers BM, Paul E, Kraybill WG, McKinnon JG, Wang HJ, Elashoff R, Faries MB and Group M: Final trial report of sentinel-node biopsy versus nodal observation in melanoma. N Engl J Med 370(7): 599609, 2014.

40 Bleicher RJ, Essner R, Foshag LJ, Wanek LA and Morton DL: Role of sentinel lymphadenectomy in thin invasive cutaneous melanomas. J Clin Oncol 21(7): 1326-1331, 2003.

41 Jacobs IA, Chang CK, DasGupta TK and Salti GI: Role of sentinel lymph node biopsy in patients with thin $(<1 \mathrm{~mm})$ primary melanoma. Ann Surg Oncol 10(5): 558-561, 2003.

42 Jacobs IA, Chevinsky AH, Swayne LC, Magidson JG, Britto EJ and Smith TJ: Gamma probe-directed lymphatic mapping and sentinel lymphadenectomy in primary melanoma: Reliability of the procedure and analysis of failures after long-term follow-up. J Surg Oncol 77(3): 157-164, 2001.

43 Chao C, Wong SL, Ross MI, Reintgen DS, Noyes RD, Cerrito PB, Edwards MJ, McMasters KM and Sunbelt Melanoma Trial G: Patterns of early recurrence after sentinel lymph node biopsy for melanoma. Am J Surg 184(6): 520-524; discussion 525, 2002.

44 Testori A, De Salvo GL, Montesco MC, Trifiro G, Mocellin S, Landi G, Macripo G, Carcoforo P, Ricotti G, Giudice G, Picciotto F, Donner D, Di Filippo F, Soteldo J, Casara D, Schiavon M, Vecchiato A, Pasquali S, Baldini F, Mazzarol G, Rossi CR and Italian Melanoma I: Clinical considerations on sentinel node biopsy in melanoma from an italian multicentric study on 1,313 patients (SOLISM-IMI). Ann Surg Oncol 16(7): 2018-2027, 2009. 
45 Nowecki ZI, Rutkowski P, Nasierowska-Guttmejer A and Ruka W: Survival analysis and clinicopathological factors associated with false-negative sentinel lymph node biopsy findings in patients with cutaneous melanoma. Ann Surg Oncol 13(12): 1655-1663, 2006.

46 Cascinelli N, Bombardieri E, Bufalino R, Camerini T, Carbone A, Clemente C, Lenisa L, Mascheroni L, Maurichi A, Pennacchioli E, Patuzzo R, Santinami M and Tragni G: Sentinel and nonsentinel node status in stage IB and II melanoma patients: Two-step prognostic indicators of survival. J Clin Oncol 24(27): 4464-4471, 2006.

47 Scoggins CR, Martin RC, Ross MI, Edwards MJ, Reintgen DS, Urist MM, Gershenwald JE, Sussman JJ, Dirk Noyes R, Goydos JS, Beitsch PD, Ariyan S, Stromberg AJ, Hagendoorn LJ and
McMasters KM: Factors associated with false-negative sentinel lymph node biopsy in melanoma patients. Ann Surg Oncol 17(3): 709-717, 2010.

48 Jones EL, Jones TS, Pearlman NW, Gao D, Stovall R, Gajdos C, Kounalakis N, Gonzalez R, Lewis KD, Robinson WA and McCarter MD: Long-term follow-up and survival of patients following a recurrence of melanoma after a negative sentinel lymph node biopsy result. JAMA Surg 148(5): 456-461, 2013.

Received September 30, 2016

Revised October 14, 2016

Accepted October 25, 2016 